\title{
A Case of Disseminated Peritoneal Tuberculosis Mimicking Metastatic Ovarian Carcinoma
}

\author{
${ }^{1}$ Nilopher Surti, ${ }^{2}$ Babu S Patel, ${ }^{3}$ Nimish C Pandya, ${ }^{4}$ Ishita Mishra
}

\begin{abstract}
Background: Mycobacterium tuberculosis affects about $30 \%$ of world's population and infection occurs at a rate of one per second. In 2010, tuberculosis accounted for 8.8 million new cases, was responsible for 1.45 million deaths with majority of cases in developing countries. Tuberculosis is the most common cause of mortality in women of reproductive age. Peritoneal tuberculosis accounts for $4 \%$ of all extrapulmonary diseases and often mimics ovarian malignancy due to its nonspecific symptoms.
\end{abstract}

Case: A 25-year-old woman with ovarian mass and an elevated cancer antigen 125 (CA-125) underwent an exploratory laparotomy to search for ovarian tumor. The final pathological diagnosis was miliary abdominal tuberculosis.

Discussion: Peritoneal tuberculosis often mimics ovarian malignancy due to its nonspecific symptoms and is a major health problem in developing countries. There is no particular diagnostic test to differentiate this disease from advanced ovarian carcinoma, making it difficult to avoid extensive surgery. Laparoscopy is the diagnostic modality of choice.

Keywords: Tuberculosis, Ascites, Ovarian carcinoma.

How to cite this article: Surti N, Patel BS, Pandya NC, Mishral. A Case of Disseminated Peritoneal Tuberculosis Mimicking Metastatic Ovarian Carcinoma. J South Asian Feder Menopause Soc 2014;2(1):44-45.

Source of support: Nil

Conflict of interest: None

\section{CASE REPORT}

A 25-year-old female patient, $\mathrm{G}_{2} \mathrm{P}_{2} \mathrm{~A}_{0} \mathrm{~L}_{2}$, presented with fever, abdominal pain and abdominal distention since 4 days. Abdominal pain was moderate in severity and generalized in nature. Her appetite was normal with no history of vomiting,

\footnotetext{
${ }^{1}$ Fellowship Student, ${ }^{2}$ Associate Professor, ${ }^{3}$ Professor and Head, ${ }^{4}$ Resident Doctor

${ }^{1}$ Department of Obstetrics and Gynecology, AV Hospital, Rajiv Gandhi University of Health Sciences, Bengaluru, Karnataka India

${ }^{2}$ Department of Obstetrics and Gynecology, Sheth VS General Hospital, Ahmedabad, Gujarat, India

${ }^{3,4}$ Department of Obstetrics and Gynecology, Smt SCL Hospital Ahmedabad, Gujarat, India
}

Corresponding Author: Nilopher Surti, Resident Doctor, Department of Obstetrics and Gynecology, Sheth VS General Hospital Ahmedabad, Gujarat, India, e-mail: nilophersurti@gmail.com diarrhea, or weight loss. She had normal menstrual cycles. Patient was a known case of noninsulin-dependent diabetes mellitus (NIDDM) and was on oral hypoglycemic agents. She had no family history of infectious or genetic diseases or any cancers.

She had an average economical background, was fairly built and nourished with no pallor, icterus, clubbing, lymph node enlargement, or pyrexia.

Abdomen was nontender on palpation with stretched out transverse umbilicus. Fluid thrill and shifting dullness were present with no hepatosplenomegaly. Bowel sounds were normal. There was a dull percussion note and decreased air entry on the right lower lung field. The rest of the respiratory examination was normal with respiratory rate of 16 per minute. Gynecological examination results were insignificant.

There was blunting of right costophrenic angle on chest $\mathrm{X}$-ray. Abdominal X-ray was normal. On ultrasound, rightsided mild pleural effusion, mild to moderate ascites, and mild splenomegaly were noted. Right ovary had a $3 \times 3 \mathrm{~cm}$ sized cystic lesion with moving internal echoes and solid component with no internal vascularity, suggesting complex right ovarian cyst. Bowel loops appeared normal.

Laboratory work up indicated mild anemia ( $\mathrm{Hb}$ : 9.5 $\mathrm{gm} / \mathrm{dl}$ ) with normal total and differential leukocyte count and mild thrombocytosis $\left(594000 / \mathrm{mm}^{3}\right)$. Her liver function test, renal function test, and serum electrolytes levels were normal. Total serum proteins were $8.2 \mathrm{gm} / \mathrm{dl}$, albumin $3.8 \mathrm{gm} / \mathrm{dl}$, and albumin to globulin ratio (A/G ratio) was 0.86 . Her CA-125 was raised $(332 \mathrm{U} / \mathrm{ml})$. Erythrocyte sedimentation rate was $40 \mathrm{~mm} / \mathrm{hr}$. She tested negative for HIV and HBsAg. Mantoux test was weakly positive. Pap test showed superficial intermediate cells with inflammatory cells consisting of neutrophils. Her urine pregnancy test (UPT) was negative. Ascitic fluid tapping was done. The tap was straw colored and revealed small mature lymphocytes with reactive mesothelial cells. It was negative for malignant cells or acid fast bacilli (AFB).

This case was referred to the gynecology unit on suspicion of Meig's syndrome in view of ovarian mass, ascites, and right-sided pleural effusion. However, ovarian involvement in this syndrome consists of a solid tumor unlike our case. In view of her symptoms, duration of illness, a negative 


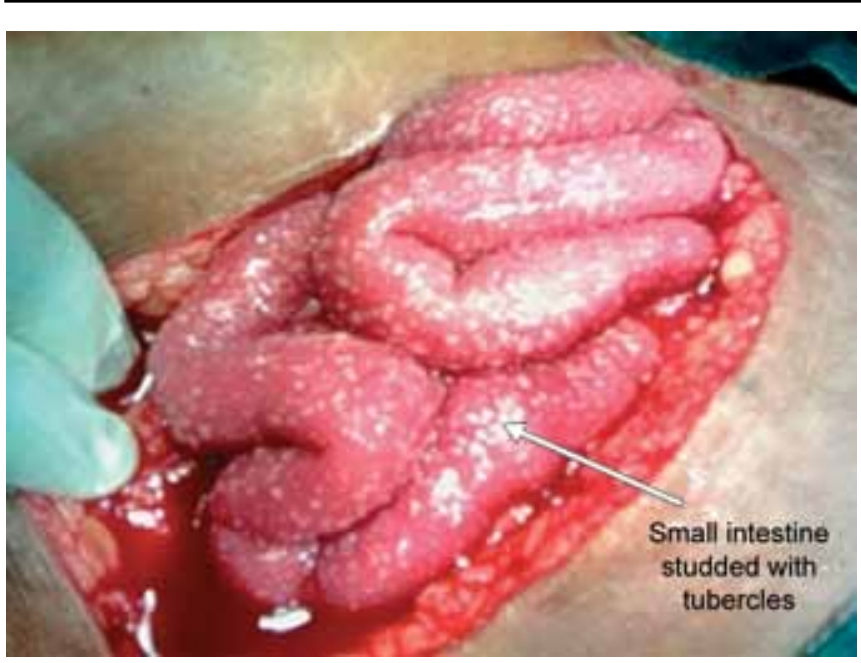

Fig. 1: Tubercles on intestinal surface

UPT, raised CA-125 and ultrasound findings, a differential diagnosis was made which included cystadenocarcinoma of the ovary and abdominal tuberculosis. Because of the inconclusive diagnosis exploratory laparotomy was planned.

Operative findings included an omental umbrella studded with approximately 2 to $3 \mathrm{~mm}$ sized tubercles. Approximately, $3 \times 3 \mathrm{~cm}$ sized right ovarian cyst was seen which aspirated. Cyst wall was excised and sent for histopathological examination along with omental biopsy. Also, $1000 \mathrm{cc}$ of ascitic fluid was drained. Multiple tubercles were seen over the surface of intestine, uterus, and adnexa (Fig. 1).

Histopathological diagnosis came out to be omental miliary tuberculosis and hemorrhagic ovarian cyst. Patient was put on anti-Kochs treatment (AKT) with intensive phase of 2 months on isoniazid, rifampicin, pyrazinamide, ethambutol (HRZE) followed by continuation phase of 4 months of isoniazid and rifampicin (HR). After 2 weeks, her ascites and pleural effusion were resolved.

\section{DISCUSSION}

Mycobacterium tuberculosis affects around 30\% of world's population. In 2010, tuberculosis accounted for 8.8 million new cases and was responsible for 1.45 million deaths, with a majority of cases in developing countries. About $80 \%$ people in Asian and African countries have tested positive in tuberculin test. Tuberculosis is the most common cause of mortality in women of reproductive age (World Health Organization). Peritoneal tuberculosis accounts for $4 \%$ cases of extrapulmonary tuberculosis. ${ }^{1}$ Its wet form presents with ascites and often has a clinical picture similar to ovarian or primary peritoneal carcinoma. Hence, it is misdiagnosed and the patient is subjected to unnecessary and extended surgery.

All investigations for diagnosing peritoneal tuberculosis have proven to be inconclusive. Only histopathologic investigation is confirmatory. ${ }^{1}$ Microbial agent can be isolated from ascitic fluid in $<50 \%$ cases. $^{2}$ The tuberculin test has only $55 \%$ sensitivity and $80 \%$ specificity, and is positive in only 40 to $85 \%$ of patients and thus, does not contribute to the diagnosis. ${ }^{1,3}$ The estimation of adenosine deaminase (ADA) levels in ascitic fluid have $98 \%$ specificity, $96 \%$ sensitivity, $88 \%$ negative predictive value, and a high positive predictive value of $95 \% .{ }^{1,3}$ Levels more than 40 units per liter are indicative of tuberculosis. Lactate dehydrogenase (LDH) assay in ascitic fluid has $90 \%$ sensitivity but only $14 \%$ specificity. ${ }^{3}$ A number of newer investigations such as enzyme-linked immunosorbent assay (ELISA) and polymerase chain reaction (PCR) have also been discussed. PCR analysis of ascitic fluid has $93 \%$ sensitivity and $84 \%$ specificity. It can be performed rapidly but is expensive and is not commonly used. ${ }^{1}$ ELISA is a simple and relatively inexpensive technique for detection of antibodies against M. tuberculosis. Its immunoglobulin G (IgG) antibody has $66 \%$ sensitivity and $81 \%$ specificity. IgM antibody has $70 \%$ sensitivity and $90 \%$ specificity. ${ }^{4}$ Imaging modalities like ultrasound and computed tomography (CT) scan have remained unsatisfactory in accurately discerning peritoneal tuberculosis. ${ }^{1}(18 \mathrm{~F})$-Fluorodeoxyglucose positron emission tomography is also useful in the imaging tuberculous lesions as fluorodeoxyglucose accumulates in inflammatory cells such as neutrophils and activated macrophages at the site of inflammation, but is very expensive. ${ }^{1,5}$ CA-125 level is a nonspecific marker as its level can increase in peritoneal tuberculosis, ovarian, primary peritoneal and other malignancies. ${ }^{1}$ However, serial CA-125 levels have prognostic value. ${ }^{1}$ When in doubt, laparoscopy is the diagnostic modality of choice, as it aids in collecting biopsy samples and avoid extensive surgery. ${ }^{1}$

\section{REFERENCES}

1. Ozan H, Ozerkan K, Orhan A. Peritoneal tuberculosis mimicking peritoneal carcinomatosis. Eur J Gynaecol Oncol 2009;30(4): 426-430.

2. Corapcioglu F, Guvenc BH, Sarper N, Aydogan A, Akansel G, Arisoy ES. Peritoneal tuberculosis with elevated serum CA 125 level mimicking advanced ovarian carcinoma in an adolescent. Turk J Pediatr 2006;48(1):69-72.

3. Mohamed M, Zainab H. A 21-year-old female with atypical presentation of TB Peritonatis. African Journals Online 2006; 15(1):40-42.

4. Omrani M, Ansari MH, Agaverdizadae D. PCR and Elisa methods (IgG and IgM): their comparison with conventional techniques for diagnosis of Mycobacterium tuberculosis. Pak J Biol Sci 2009;12(4):373-377.

5. Alavi A, Gupta N, Alberini JL, Hickeson M, Adam LE, Bhargava P, Zhuang H. Positron emission tomography imaging in nonmalignant thoracic disorders. Semin Nucl Med 2002; 32(4):293-321. 